\title{
Análise e Tratamento da Informação
}

Lúcia Santos*

\section{RESUMO}

O presente artigo aborda os conceitos gerais relacionados com a pesquisa bibliográfica, no contexto da investigação conducente à produção de conhecimento.

Foca, em particular, a pesquisa bibliográfica em bases de dados electrónicas enquanto procedimento metodológico para a revisão do conhecimento científico, descrevendo, resumidamente, cada uma das principais bases de dados electrónicas, na área das ciências da saúde.

Adicionalmente, refere alguns conceitos para a elaboração de uma estratégia de pesquisa, descrevendo os principais recursos disponíveis para operar os sistemas de pesquisa de forma optimizada.

Palavras-Chave: Pesquisa Bibliográfica; Bases de Dados Electrónicas; Procedimentos Metodológicos.

\section{INTRODUÇÃO}

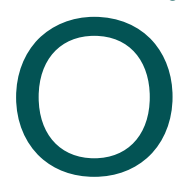

recurso a técnicas de pesquisa bibliográfica, em bases de dados electrónicas, é um requisito fundamental no âmbito de um trabalho de revisão da literatura científica.

O desenvolvimento de um trabalho de investigação deverá iniciar-se, em qualquer circunstância, pela revisão da evidência científica já produzida, no âmbito do tema em estudo.

De facto, um adequado e correcto trabalho de revisão é um requisito fundamental para o enquadramento do problema e para definir e orientar, objectivamente, a questão a investigar.

Este trabalho de revisão da literatura científica assenta, fundamentalmente, na pesquisa em bases de dados electrónicas, devendo o investigador dominar, ainda que de forma genérica, as técnicas e os métodos desta pesquisa.

Neste artigo é revista a metodologia para a definição de uma estratégia de pesquisa, sublinhando-se a necessidade de um correcto enquadramento teórico e da correcta definição conceptual do problema, enquanto pontos prévios que determinam a eficiência da pesquisa.

Abordam-se, igualmente, as principais bases de dados electrónicas, na área das ciências da saúde, bem como as características de cada uma.

*Farmacêutica, Mestre em Saúde Pública
Descreve-se, por fim, a metodologia para a elaboração de uma correcta equação de pesquisa, referindo-se, concretamente, a utilização dos operadores boleanos e de outras técnicas, que possibilitam uma maior especificidade dos resultados da pesquisa.

\section{FONTES DE INFORMAÇÃO}

As fontes de informação podem classificar-se como primárias, secundárias ou terciárias. Embora esta classificação possa não ser absolutamente concordante nos diversos ramos da ciência, existindo ligeiras diferenças nos critérios de classificação de algumas fontes, nomeadamente entre as ciências exactas e as ditas humanidades, podemos genericamente adoptar as seguintes definições: ${ }^{1-3}$

- As fontes primárias incluem todo o tipo de informação original, que não tenha sido alvo de processos de interpretação e/ou de avaliação. Constituem, normalmente, a primeira apresentação de um pensamento original, do relatório de uma nova descoberta ou de informação nova acerca de um determinado assunto.

Como exemplos de fontes primárias destacam-se os artigos científicos publicados em revistas da especialidade, nas quais a informação surge pela primeira vez, as patentes ou proceedings de reuniões e conferências.

- As fontes secundárias reportam-se a todo o tipo de 
material e informação produzida a partir de fontes primárias, originais. A informação contida nas fontes secundárias foi normalmente alvo de modificação, selecção ou reorganização no sentido de servir um objectivo determinado, ou para se adequar a um público específico. Constituem, normalmente, interpretações e/ou avaliações de fontes primárias.

Os exemplos mais relevantes deste tipo de fonte são as monografias, os artigos de revisão, os manuais e os livros de texto, os dicionários e enciclopédias.

- As fontes terciárias são mais difíceis de definir, tanto mais que se sobrepõem, com frequência, aos conteúdos normalmente considerados como fontes secundárias.

Genericamente, podemos considerá-las como fontes indirectas de informação. São, normalmente, sistematizações ou referências de fontes primárias e secundárias, como sejam as revistas de resumos, os índices cumulativos periódicos, as listas bibliográficas, entre outros.

Num trabalho de revisão sistemática da bibliografia sobre um determinado tema, devemos, preferencialmente, iniciar a pesquisa nas fontes secundárias de informação, de forma a construirmos, desde logo, uma visão mais abrangente do assunto e enquadrarmos, o melhor possível, a questão a investigar., ${ }^{2,4}$

Ultrapassada esta etapa, torna-se fundamental pesquisar as fontes primárias, no sentido de obter a informação inédita e mais actual sobre o assunto em investigação.

Esta etapa, a de identificar os artigos de maior relevo no meio das dezenas de milhar publicados anualmente, exige, naturalmente, a utilização de adequadas ferramentas de pesquisa. Hoje em dia, estas ferramentas são, quase em exclusivo, bases de dados electrónicas, que permitem, através da utilização de palavras-chave e adequadas equações de pesquisa, a selecção de um conjunto de artigos que preenchem os requisitos formulados.

\section{O DESENVOLVIMENTO DE UMA ESTRATÉGIA DE PESQUISA}

O desenvolvimento de uma metodologia para a revisão sistemática da evidência científica sobre um determinado tema deve iniciar-se, em qualquer circunstância, pelo estabelecimento de um protocolo de trabalho..$^{4-6}$
O protocolo deve definir, com clareza, os seguintes aspectos: ${ }^{4,6}$

- Questão a ser revista;

- Critérios de inclusão e de exclusão na pesquisa;

- Identificação de estudos potencialmente relevantes;

- Selecção de estudos relevantes;

- Extracção de dados.

Nesta última etapa, deverá ser avaliada a qualidade dos estudos e elaborado o resumo da evidência científica.

Deverá ser dada uma atenção especial à formulação do problema a ser revisto na pesquisa, sendo fundamental fazê-lo de forma precisa e específica. Os aspectos mais laterais ao problema derivam, naturalmente, da questão original.

Também se compreende que, frequentemente, uma melhor compreensão do problema em estudo é adquirida no decurso do processo de revisão da literatura, pelo que a questão poderá ser reformulada durante $o$ mesmo.

\section{A IDENTIFICAÇÃO DE LITERATURA RELEVANTE}

A hierarquização do tipo de literatura disponível, no que respeita à sua importância para a investigação, depende, frequentemente, do tipo de estudo e da área científica em causa.

Na área da saúde, e da medicina em particular, importa, sobretudo, valorizar as intervenções que assentam no princípio da medicina baseada na evidência, na medida em que é fundamental avaliar a eficácia das intervenções clínicas. ${ }^{6}$

Para este propósito, existem alguns tipos de estudo que permitem a determinação, com maior sustentabilidade, de relações de causalidade, sendo, normalmente, adoptada a seguinte hierarquia de desenhos de estudo:, 7

- Experimental (estudos de intervenção)

Meta-análises de Ensaios Clínicos Randomizados

Ensaios Clínicos Randomizados

Estudos quasi-experimentais (não randomizados)

- Observacional

Estudos de Coorte

Estudos de Caso-Controlo

Estudos transversais

Estudos ecológicos

Nesta hierarquia, a primazia é dada aos ensaios clí- 
nicos randomizados (ECR). A randomização, em estudos intervencionados, permite evitar os viéses de confundimento, na medida em que um ECR correctamente desenhado garante que a única diferença existente entre o grupo intervencionado e o grupo de controlo é a própria intervenção. ${ }^{6,7}$

Este pressuposto não deve, contudo, em nenhuma circunstância, ditar a exclusão de qualquer tipo de evidência na condução de uma pesquisa. Todo o tipo de estudo deverá ser considerado, sendo que o investigador deverá ter a capacidade de fazer uma avaliação crítica dos mesmos.

Na verdade, existe um conjunto de problemas de natureza prática, e inerentes aos próprios ECR's, que fazem com que este não seja o tipo de estudo ideal como fonte de evidência para responder a todo o tipo de questões biológicas. ${ }^{6}$

O consenso que tem vindo a ser construído acerca da inferência de causalidade requer que esta seja estabelecida com uma base inequívoca na evidência, num contexto biológico amplo.

Alguns dos factores normalmente utilizados para estabelecer uma relação causal entre uma exposição e um outcome e, portanto, determinar relações de causalidade, foram claramente definidos por Bradford Hill e Rothman. ${ }^{6,7}$

Destacamos, entre eles, a força, ou poder, da associação estabelecida, a sua consistência, existência de uma relação dose-resposta, a plausabilidade biológica e a especificidade da associação. Estas características são importantes para concluir sobre a causalidade de uma associação, embora não possam ser considerados como critérios rígidos.

Não existem pré-requisitos absolutos nesta matéria, sendo que não é obrigatória a aplicação de todos os critérios, em todas as circunstâncias. Em algumas circunstâncias, poderá ser mais relevante a informação proveniente de estudos observacionais do que de ECR's, devendo o investigador fazer uma avaliação crítica da qualidade da informação disponível e concluir sobre a adequação do tipo de estudo à questão que está a ser abordada.

Para avaliar a qualidade global de um trabalho, devemos levar em consideração aspectos como a sua relevância clínica, o desenho do estudo, a forma como foi conduzido, a análise dos dados e a qualidade da pró- pria redacção. ${ }^{6}$

No seu conjunto, estes aspectos vão fornecer informação acerca da validade, interna e externa, dos resultados.

A validade interna relaciona-se, fundamentalmente, com o grau de minimização dos viéses no trabalho, o que depende da sua arquitectura, designadamente de aspectos como o desenho, a condução e a análise de dados do estudo. ${ }^{6,7}$

A validade externa relaciona-se com a extensão na qual é possível generalizar os resultados, ou seja, a sua reprodutibilidade para além das fronteiras do trabalho. Neste caso, os aspectos que mais importam são a população estudada, a intervenção / exposição, os métodos utilizados e os outcomes. ${ }^{6,7}$

\section{BASES BIBLIOGRÁFICAS}

Existem numerosas bases de dados, electrónicas, que nos permitem aceder, com grande rapidez e facilidade, a um conjunto de referências bibliográficas de interesse e, em alguns casos, ao texto integral dos trabalhos publicados.

A pesquisa manual não se justifica hoje em dia, excepto nas situações em que é importante procurar artigos não identificados pela pesquisa electrónica, ou fazer a pesquisa em publicações periódicas relevantes, não incluídas nas bases de dados utilizadas.

O factor que, provavelmente, mais determinará o sucesso da pesquisa é a capacidade do investigador em delimitar o conteúdo pretendido, bem como em identificar uma, ou um conjunto de palavras-chave, que permitam que a resposta nos forneça toda a informação de relevo publicada sobre o tema. Estas palavras-chave devem, ao mesmo tempo, excluir, desde logo, toda a informação que, pese embora possa relacionar-se com o assunto em causa, não seja relevante para a investigação que se pretende. Encurta-se, deste modo, o sempre laborioso trabalho de triagem da lista bibliográfica devolvida pela base.

As palavras-chave seleccionadas deverão, preferencialmente, ser em língua inglesa, atendendo a que a grande maioria da informação científica é publicada nesta língua, ou tem resumos e palavras-chave neste idioma.

Existem numerosas bases de dados para a pesquisa bibliográfica, muitas delas genéricas e outras dedica- 
das, preferencialmente, a uma área específica do conhecimento científico.

É aconselhável, e mesmo necessária, a pesquisa em várias bases de dados diferentes, no sentido de assegurar a devolução de uma lista de referências consistente e o mais completa possível. Na verdade, cada base de dados subscreve a sua própria lista de publicações, sendo, por vezes, baixa a percentagem de sobreposição de títulos, mesmo entre as principais bases.

Existem algumas bases de dados, vocacionadas para as áreas da medicina e das ciências da saúde, que, consensualmente, se consideram como o conjunto mínimo recomendado de bases a pesquisar, para iniciar qualquer trabalho de pesquisa bibliográfica., ${ }^{8,9}$

Faremos uma breve descrição de cada uma delas.

\section{MEDLINE}

A base MEDLINE ${ }^{\circledR}$ (sigla da expressão inglesa Medical Literature and Retrieval System Online) é a principal base de dados publicada pela Biblioteca Nacional de Medicina dos Estados Unidos da América (US National Library of Medicine), contendo citações e resumos de trabalhos de, aproximadamente, 5.000 publicações da área biomédica, de todo o mundo. ${ }^{9-11}$

Actualmente, possui mais de 16 milhões de referências bibliográficas, com datas tão anteriores quanto 1949, fornecendo, em alguns casos, para além da referência, informações adicionais, tais como o resumo do trabalho (aproximadamente $79 \%$ das citações incluem o abstract) e o endereço do autor para correspondência. $^{11}$

Cada citação MEDLINE ${ }^{\circledast}$ representa um artigo e contém campos que fornecem informação diversa acerca do artigo. Normalmente, é fornecida a seguinte informação: ${ }^{11}$

- Título do artigo;

- Nome do(s) autor(es);

- Resumo do trabalho publicado com o artigo;

- Termos de pesquisa controlados (vocabulário $\mathrm{MeSH})$;

- Afiliação do primeiro autor;

- Idioma no qual o artigo está publicado;

- Tipo de publicação (artigo original, revisão de conjunto,...)

A base é actualizada diariamente, de terça-feira a sábado. Embora tenha uma cobertura mundial, a maio- ria dos registos (cerca de 90\%) são em língua inglesa, ou têm um resumo neste idioma. ${ }^{11}$

\section{PUBMED $^{\circ}$}

A PubMed ${ }^{\circledast}$ é uma base de dados desenvolvida pelo Centro Nacional para a Informação Biotecnológica (NCBI - National Center for Biotechnology Information) na US National Library of Medicine, de acesso gratuito.-11

Esta base de dados, que inclui cerca de 17 milhões de citações bibliográficas ${ }^{11}$, é uma das interfaces de pesquisa na MEDLINE ${ }^{\circledR}$, permitindo, igualmente, o acesso a outras publicações periódicas da área das ciências da vida e da biomedicina.

A PubMed ${ }^{\circledR}$, que utiliza o motor de busca Entrez, inclui links para acesso ao texto integral de muitos artigos, a partir dos sites dos respectivos editores, e disponibiliza outros recursos relacionados, utilizando o vocabulário MeSH. ${ }^{11}$

A base de dados PubMed ${ }^{\oplus}$ disponibiliza um conjunto de serviços de grande utilidade para o investigador. ${ }^{11}$

- Journals Database: base de dados de publicações periódicas;

- MeSH Database: base de dados de vocabulário $\mathrm{MeSH}$;

- Single Citation Matcher: permite a localização de um artigo específico;

- Batch Citation Matcher: é uma ferramenta para os editores;

- Clinical Queries: página desenhada para médicos, que inclui filtros de pesquisa;

- Special Queries: dá acesso a uma directoria de questões relacionadas especificamente com a PubMed.

- LinkOut: possibilita aos utilizadores da PubMed aceder a um conjunto alargado de recursos online, incluindo o texto integral de algumas publicações;

- My NCBI: permite ao investigador guardar estratégias de pesquisa já formuladas e obter actualizações automáticas sobre o tema.

\section{Vocabulário MeSH}

MeSH é o acrónimo da expressão inglesa Medical Subject Headings, e é usada para indexar artigos para a base MEDLINE ${ }^{\circledR} /$ PUBMED. É o equivalente às palavras-chave noutros sistemas de pesquisa. ${ }^{11}$

A utilização deste vocabulário controlado, revisto anualmente, permite uniformizar e dar consistência à 
indexação de literatura biomédica, e é uma das características particulares da MEDLINE ${ }^{\circledR}$.

MeSH é, assim, a designação dada à base de dados que assiste a PubMed na identificação de termos adequados de pesquisa. A base de dados inclui informação sobre os termos MeSH, incluindo a sua definição, sinónimos do conceito e termos relacionados.

\section{EMBASE ${ }^{\circledR}$}

A EMBASE ${ }^{\circledast}$ é uma base de dados desenvolvida pela Elsevier, uma das maiores editoras de literatura médica e científica do mundo, com sede em Amsterdão. É uma fonte de informação privilegiada nas áreas da farmacologia e da biomedicina, útil pela extensa indexação de informação sobre fármacos e medicamentos, de 4.550 publicações oriundas de 70 países. ${ }^{910,13}$

Cada registo nesta base de dados contém a citação bibliográfica, os termos de indexação e os códigos associados, e mais de $80 \%$ dos registos incluem resumos do trabalho. ${ }^{7,9,10}$

Os registos da EMBASE ${ }^{\circledR}$ são indexados usando a EMTREE, uma base de dados de vocabulário controlado desenvolvido pela Elsevier, com cerca de 55.000 entradas e mais de 200.000 sinónimos. $^{13}$

$\mathrm{O}$ acesso é feito mediante assinatura.

\section{SCOPUS}

Igualmente produzida pela Elsevier, a Scopus tem vindo a ganhar uma notoriedade enorme nos últimos anos. É, hoje em dia, a maior base de dados de referências e abstracts, cobrindo mais de 16.000 revistas com arbitragem, de cerca de 4.000 editores. ${ }^{14}$

Para além de contemplar todas as publicações indexadas pela PubMed, a Scopus possibilita, ainda, a pesquisa das referências citadas nos artigos publicados a partir de 1996, bem como aceder ao número de vezes que cada artigo foi citado por outros, igualmente indexados pela base. ${ }^{14}$

Estas características eram, até muito recentemente, exclusivas da Web of Science, produzida pelo ISI - Institute for Scientific Information.

\section{ISI WEB OF KNOWLEDGE}

É um portal desenvolvido nos Estados Unidos pelo ISI - Institute for Scientific Information que agrega bases de dados multidisciplinares, contendo informação de di- ferentes áreas científicas. ${ }^{9,10,15}$ A plataforma ISI Web of Knowledge oferece acesso integrado a um conjunto de recursos, dos quais destacamos a pesquisa na base Web of Science ${ }^{\circledR}$.

A Web of Science é a designação corrente dada a um conjunto de bases de dados, de natureza multidisciplinar, também conhecidas como «Science Citation Indexes», ${ }^{15}$ compiladas pelo ISI.

Incluem-se a «Science Citation Index», a «Social Science Citation Index» e a "Arts and Humanities Citation Index». ${ }^{15}$

São bases de natureza referencial que possuem como característica distintiva a possibilidade de visualizar os artigos citados por um determinado artigo, ou verificar o número de vezes que determinado artigo foi citado e por quem.

É a partir destas bases que se calcula o factor de impacto das publicações periódicas, bem como outros indicadores bibliométricos referenciados no «Journal Citation Reports», base de dados que disponibiliza informação estatística que permite avaliar o impacto e a disseminação das publicações na comunidade científica. ${ }^{15}$

A pesquisa na Web of Science, através da interface ISI Web of Knowledge, possibilita a pesquisa simultânea nas diversas bases de dados que integram a plataforma. O resultado da pesquisa, para cada registo, inclui o título, o(s) nome(s) do(s) autor(es) e a fonte, possibilitando igualmente a visualização do abstract do documento, os dados de citação e a possibilidade de pesquisa dos registos relacionados.

Os dados de citação são o elemento que distingue esta de outras bases de dados de referência.

\section{BIOSIS (Previews)}

Indexa informação de mais de 6.000 revistas das áreas da saúde e da biologia, e também de outras fontes, tais como livros, patentes ou encontros científicos. ${ }^{9,10}$

\section{SciSearch}

É uma base de dados multidisciplinar. Para além de todos os registos indexados no Science Citation Index, integra, adicionalmente, registos na área da engenharia tecnológica, física, agricultura, biologia, ciências ambientais e ciências da vida, no geral. Indexa todos os items relevantes (papers, artigos de revisão, abstracts de reuniões científicas, cartas, editoriais, comentários crí- 
ticos a obras, etc) de mais de 6.100 publicações técnicas e científicas internacionais. ${ }^{9,10}$

\section{PASCAL}

Trata-se de uma base de dados multidisciplinar, desenvolvida em França. Pese embora se defina como uma base de abrangência internacional, dá primazia às publicações francesas, incluindo teses e dissertações. ${ }^{9}$

Os registos são indexados numa versão trilingue possibilitando que, independentemente do idioma do artigo, a pesquisa possa ser efectuada em inglês, francês ou espanhol. ${ }^{16}$

\section{LILACS}

É uma base de dados bibliográfica criada em 1985 para o registo de literatura técnica e científica, na área das ciências da saúde, desenvolvida e produzida pelos países da América Latina e do Caribe. ${ }^{9}$

Indexa, entre outros, artigos de publicações periódicas, livros e monografias, teses, manuais, relatórios técnico-científicos e outros documentos não convencionais.

O maior contribuidor para esta base de dados é o Brasil, cuja literatura indexada constitui mais de metade do total de registos inseridos no LILACS. ${ }^{9}$

\section{CAB ABSTRACTS}

É uma base de dados de âmbito multidisciplinar, desenvolvida em Inglaterra ${ }^{9}$, contendo mais de 4 milhões de registos provenientes de cerca de 11.000 fontes, incluindo revistas, livros, conferências, relatórios e outro tipo de literatura publicada internacionalmente. ${ }^{17}$

Esta base indexa registos publicados desde 1973, com abstracts em inglês preparados a partir de trabalhos publicados originalmente em 75 línguas diferentes. $^{17}$

\section{COCHRANE LIBRARY incorporating}

Em 1972, Archie Cochrane, um epidemiologista britânico, chamou a atenção para a necessidade de os médicos disporem de revisões sistemáticas, periodicamente actualizadas, sobre a evidência científica disponível nas diversas áreas. ${ }^{18}$

A Cochrane Collaboration surgiu em resposta ao apelo de Cochrane, designadamente da necessidade de disponibilização de revisões sistemáticas dos ensaios clínicos randomizados relevantes, tendo o primeiro Centro Cochrane surgido em Oxford, em 1992.

Hoje, é uma rede que envolve cerca de 4.000 pessoas que trabalham, maioritariamente, numa base de voluntariado, produzindo e actualizando revisões sistemáticas, na área dos ensaios clínicos.

Como princípio de base, a Cochrane assume que o cerne do seu trabalho assenta na medicina baseada na evidência (MBE), sendo que a sua biblioteca reflecte justamente este princípio.

O conceito de níveis de evidência é central na MBE, e também na estrutura da biblioteca Cochrane..$^{19}$ Os melhores tipos de evidência são conferidos, por ordem decrescente de importância, por:

1. Meta-análise de bons ECR's

2. Um ECR de boa qualidade

3. Um estudo controlado, bem desenhado, com algum nível de randomização

4. Um estudo controlado, bem desenhado, sem randomização

Este conceito reflecte-se nas diferentes bases de dados da Cochrane Library:

- Base de Dados DARE (Database of Abstracts of Reviews of Effectiveness)

Inclui resumos estruturados de revisões sistemáticas, de todo o mundo, previamente avaliados por especialistas da Universidade de York.

\section{- Base de Dados de revisões sistemáticas}

É um conjunto, crescente, de revisões sistemáticas, regularmente actualizadas, essencialmente de ECR's. A evidência é considerada na base de critérios de qualidade explícitos, para minimizar os viéses. Os dados são sempre combinados estatisticamente para produzir meta-análises e, desta forma, permitir a inclusão de estudos demasiado pequenos para poderem produzir resultados fiáveis, isoladamente.

\section{- Base CCTR (Cochrane Controlled Trials Register)}

Trata-se de uma lista bibliográfica de ensaios controlados, identificados pelos contribuidores. A base inclui relatórios publicados em livros de resumos de conferências e congressos e outros registos que, habitualmente, não são incluídos noutras bases de dados.

No âmbito de uma assinatura em consórcio, o conjunto de bases de dados da Cochrane Library está disponível em todos os hospitais portugueses que integram o consórcio nacional «B-on-Biblioteca do Conhe- 
cimento Online», com acesso através da plataforma EBSCO.

\section{HTA}

A base de dados HTA contém registos (projectos e publicações) sobre avaliação de tecnologias em saúde. É produzida pelo CRD/NHS (Centre for Reviews and Dissemination of the National Institute for Health Research), um departamento da Universidade de York, usando informações obtidas dos membros do INAHTA (International Network of Agencies for Health Techno$\operatorname{logy}$ Assessment). O INAHTA é uma organização sem fins lucrativos, estabelecida para promover a cooperação e a partilha de informação entre organizações de todo o mundo, que avaliam tecnologias em saúde. ${ }^{20}$

\section{A EQUAÇÃO DE PESQUISA}

Para fazer uma pesquisa numa base de dados electrónica, é importante conhecer e saber usar os recursos disponíveis, para operar o sistema de forma optimizada e elaborar uma estratégia de pesquisa que permita a identificação dos trabalhos.

Os critérios para a pesquisa bibliográfica são, essencialmente, a relevância e a amplitude. O levantamento amplo é mais abrangente, identificando muitos trabalhos, requerendo esta técnica um processo de selecção mais preciso, e uma triagem mais aturada das referências.

A escolha do tipo de levantamento depende, sobretudo, do número de artigos disponíveis sobre o assunto. Para um tema muito estudado, o levantamento deverá ser mais específico, enquanto, para um tema pouco estudado, deverá ser mais abrangente.

A especificidade ou abrangência desejada é obtida através da utilização adequada dos operadores boleanos ou, ainda, de técnicas para a pesquisa de termos que contenham caracteres desconhecidos ou múltiplas formas de serem apresentados. ${ }^{12,21}$

\section{Operadores boleanos}

\section{Operador boleano OR}

Este operador utiliza-se para agrupar termos, permitindo-nos ampliar a pesquisa. ${ }^{12,21}$ Deverá ser utilizado, por exemplo, nas seguintes circunstâncias:

a) Sinónimos: cancro OR tumor OR neoplasia

b) Acrónimos: doença pulmonar obstrutiva crónica $\mathbf{O R}$

\section{DPOC}

c) Diferentes grafias: raios $X$ OR raios-X $\mathbf{O R}$ raio $X$ OR raio-X

\section{Operador boleano AND}

É utilizado para restringir a pesquisa, fazendo uma selecção dos trabalhos que possuam os termos combinados. ${ }^{12,21}$

Assim, por exemplo, para estudar o efeito preventivo da circuncisão masculina na infecção por HIV, deverá utilizar-se:

- HIV AND circuncisão masculina

\section{Operador boleano NOT}

O operador NOT combina termos no sentido de o resultado não conter o(s) termo(s) que se associa(m) à direita do operador. ${ }^{12,21}$

\section{Truncagem}

A truncagem utiliza-se para pesquisar todas as formas derivadas ou plurais de uma determinada palavra, inserindo-se a raiz da palavra seguida do símbolo utilizado para a truncagem. ${ }^{10,21}$

Nas principais bases de dados aqui referenciadas, como a PubMed, a EMBASE ou a Web of Science, o símbolo utilizado para a truncagem é o asterisco «*», embora outras bases de dados adoptem símbolos diversos para a operação de truncatura, como é o caso da LILACS que utiliza o cifrão «\$».22

Assim, utiliza-se por exemplo: imunossupres*/imunossupres $\$$ para pesquisar imunossupressão, imunossupressor, imunossupressores, etc. Ou random*/random\$ para randomização, randomizado, etc.

\section{Wildcard}

Algumas bases de dados possibilitam a pesquisa com wildcard, através da inserção do símbolo correspondente (que poderá ser um ponto de interrogação «?» ou um asterisco «*») no meio de uma palavra a pesquisar. A base devolve os resultados substituindo automaticamente o símbolo por uma letra. ${ }^{21}$

A pesquisa com «wom?n»/«wom*n», por exemplo, devolverá os registos woman e women.

\section{CONCLUSÃO}

A pesquisa bibliográfica realiza-se, normalmente, com 
o objectivo de fundamentar teoricamente uma tese, contribuindo com elementos que subsidiam o objecto em estudo. Vai, portanto, para além da simples revisão bibliográfica, que se limita à observação dos dados contidos nas fontes pesquisadas.

Pelo contrário, o trabalho de pesquisa requer avaliação crítica da informação, sublimando um conjunto de hipóteses que irão servir à orientação da própria investigação.

A condução de um trabalho de pesquisa bibliográfica exige, assim, a definição de critérios objectivos e a delimitação clara do objecto de estudo.

Pese embora este objecto de estudo possa ser redefinido, em função do decurso do próprio trabalho de pesquisa, importa que, em cada momento, o conceito esteja clarificado, para que sejam eficazes os procedimentos metodológicos adoptados.

Da facilidade com que se obtém informação através de bases de dados electrónicas não se poderá inferir, contudo, que o trabalho de pesquisa seja simples.

Pelo contrário, é exigida disciplina e assertividade no percurso metodológico definido, bem como capacidade de análise crítica dos resultados.

\section{ENDEREÇO PARA CORRESPONDÊNCIA}

Lúcia Santos

Unidade de Avaliação de Tecnologias em Saúde do AIBILI

Azinhaga St. Comba, 3000 - 354 Coimbra

E-maiL: luciamlsantos@gmail.com

\section{REFERÊNCIAS BIBLIOGRÁFICAS}

1. BadkeWB.Welcome to the information fog. In: Research Strategies: finding your way through the information fog. 3th ed. Lincoln, NE: iUniverse.com; 2008. p. 1-14.

2. Campana AO. Metodologia da investigação científica aplicada à area biomedica: 1. O método científico. J Pneumol 1999 jan-fev; 25 (1): 25 $-34$.

3. Jones RG. Personal computer software for handling references from CDROM and mainframe sources for scientific and medical reports. BMJ 1993 Jul 17; 307 (6897): 180-4.

4. Lima TC, Mioto RC. Procedimentos metodológicos na construção do conhecimento científico: a pesquisa bibliográfica. Rev. Katálysis 2007; 10 (n. ${ }^{\circ}$ esp.): 37-45.

5. Brown JD. Power Research: who needs it? Research Strategies 1997; 15 (4): 239-60.

6. World Cancer Research Fund / American Institute for Cancer Research. Food, Nutrition, Physical Activity, and the Prevention of Cancer: a Global Perspective. Washington DC:AIRC, 2007. Chap. 3. p. 48-62. Disponível em: http://www.dietandcancerreport.org/ [acedido em 15/01/2009].
7. Strom BL. Study design available for pharmacoepidemiology studies. In: Strom BL, editor Pharmacoepidemiology. 4th ed. West Sussex: John Wiley \& Sons; 2007. p. 17-28.

8. Badke WB. Library Catalogs and Journal Databases. In: Research Strategies: finding your way through the information fog. 3th ed. Lincoln, NE: iUniverse.com; 2008. p. 71-95.

9. Coimbra CE Jr. Produção científica em saúde pública e as principais bases bibliográficas internacionais. Cad Saúde Pública 1999 out-dez; 15(4): 883-8.

10. Volpato ES. Pesquisa bibliográfica em ciências biomédicas. J Pneumol 2000 mar-abr; 26 (2): 77-80.

11. National Library of Medicine. NLM ${ }^{\circledR}$ Training: PubMed ${ }^{\circledR}$. October 2008 Revision. Disponível em: http://www.nlm.nih.gov/pubs/manuals/pm_workbook.pdf [acedido em 22/12/2008].

12. National Library of Medicine. NLM® ${ }^{\circledR}$ Training: NLM Gateway and ClinicalTrials.gov. September 2008 Revision. Disponível em: http://www. nlm.nih.gov/pubs/manuals/gwclintrials.pdf [acedido em 22/12/2008].

13. Elsevier. EMBASE Description. 2008. Disponível em: http://www.elsevier.com/wps/find/bibliographicdatabasedescription.cws_home/ 523328/description. [acedido em 15/01/2009].

14. Elsevier. SCOPUS. 2009. Disponível em: http://www.scopus.com/scopus/home.url. [acedido em 15/01/2009].

15. Thomson Reuters. ISI Web of KnowledgeSM. 2009. Disponível em: http://isiwebofknowledge.com/currentuser_wokhome/cu_aboutwok/. [acedido em 15/01/2009].

16. Ovid Technologies, Inc. PASCAL. 2009. Disponível em: http://www.ovid. com/site/catalog/DataBase/214.jsp. [acedido em 15/01/2009].

17. Ovid Technologies, Inc. CAB Abstracts. 2009. Disponível em: http://www.ovid.com/site/catalog/DataBase/31.jsp. [acedido em 15/01/2009].

18. The Cochrane Collaboration. Archie Cochrane: the name behind the Cochrane Collaboration. Disponível em: http://www.cochrane.org/docs/archieco.htm. [acedido em 15/01/2009].

19. The Cochrane Collaboration. Evidence-based medicine and health care. Disponível em: http://www.cochrane.org/docs/ebm.htm. [acedido em 15/01/2009].

20. Wiley InterScience. Product Descriptions. 2008. Disponível em: http://www3.interscience.wiley.com/cgi-bin/mrwhome/106568753/ ProductDescriptions.html\#hta. [acedido em 15/01/2009].

21. Badke WB. Database Searching with keywords and hierarchies. In: Research Strategies: finding your way through the information fog. 3th ed. Lincoln, NE: iUniverse.com; 2008. Chap.3. p. 32-52.

22. BIREME/OPAS/OMS - Centro Latino-Americano e do Caribe de Informação em Ciências da Saúde. Pesquisa em Bases de Dados - LILACS. Disponível em: http://bases.bireme.br/cgi-bin/wxislind.exe/iah/cys/ ?lsisScript $=$ iah/iah.xis\&base $=$ LILACS\&lang=p. [acedido em 15/01/2009]. 


\section{ABSTRACT}

The present article focus on the general concepts regarding the bibliographic research within the investigation context that leads to the production of knowledge.

It refers to the bibliographic research in electronic databases, as a methodological procedure for the acknowledgment of the scientific literature, briefly describing each of the main electronic databases within the Health Sciences field.

Moreover, this work systematizes the main steps to the construction of a research strategy, by describing the available key resources to operate the research systems in an optimized way.

Keywords: Bibliographic Research; Electronic Databases; Methodological Procedures. 